\title{
Mixed-Integer Nonlinear Model for Multiproduct Inventory Systems with Interior Point and Branch and Bound Method
}

\author{
A. M. Lourenção, E. C. Baptista, E. M. Soler, F. B. Souza and A. C. Cherri
}

\begin{abstract}
Inventory management can be considered as one of the main components of planning and production control. In the academic literature numerous mathematical models are presented for inventory management, which refer to different aspects related to this management. The development of efficient inventory models and the adoption of appropriate optimization methods for solving these models are needed to support in making decisions to inventory management. In this paper, we propose an inventory model that works with multiple products and multiple resource constraints, which explores the concept of continuous review and periodic review. This model is formulated as a mixed integer nonlinear optimization problem. It explores for the resolution of this model, an approach based on Branch and Bound method and interior point method. In order to propose this model and choose the method for its resolution, initially makes an investigation in the literature review on the topic. Then, it explores the concept of continuous review and periodic review. Finally, two computational tests are proposed, it one to compare the results of proposed model with the linear model and the other to verify their efficiency and applicability. The results show the potential of model and solution method to work with inventory system.
\end{abstract}

Keywords - Multiproduct Inventory Systems, Continuous Review, Periodic Review and Optimization.

\section{INTRODUÇÃO}

A GESTÃo de estoques é, tradicionalmente, um dos principais componentes do planejamento e controle da produção e envolve uma série de conceitos relativos a processos, custos, decisões e políticas que, além de permitir um melhor entendimento do funcionamento de um sistema produtivo é um importante instrumento de vantagem competitiva [1]. Alguns trabalhos, como o apresentado por [2], indicam uma relação entre a gestão de estoque e o desempenho financeiro da organização. Os autores de [3] relatam a respeito de algumas pesquisas que mostram evidências que existe um efeito em cadeia positivo entre o desempenho do estoque e a utilização de outras práticas de

A. M. Lourenção, Universidade Estadual Paulista (UNESP), Bauru, SP, Brasil, alvarodml@yahoo.com.br

E. C. Baptista, Universidade Estadual Paulista (UNESP), Bauru, SP, Brasil, baptista@fc.unesp.br

E. M. Soler, Universidade Estadual Paulista (UNESP), Bauru, SP, Brasil,edilaine@fc.unesp.br

F. B. Souza, Universidade Estadual Paulista (UNESP), Bauru, SP, Brasil, fbernardi@feb.unesp.br

A. C. Cherri, Universidade Estadual Paulista (UNESP), Bauru, SP, Brasil, adriana@fc.unesp.br manufatura, com consequente melhoria do desempenho de outras áreas.

A literatura apresenta inúmeros modelos matemáticos voltados para a gestão de estoques. Esses modelos referem-se a diversos aspectos relacionados ao controle de estoque. Os diferentes ambientes de negócios no qual as empresas estão inseridas, e as características de produção e distribuição também são fatores que explicam essa diversidade de modelos.

Um dos contextos abordados nesses modelos são os sistemas de estoque de múltiplos produtos, denominados na literatura como modelos de estoque multiprodutos. Modelos multiproduto foram desenvolvidos por vários autores como [4], [5], [6], [7] e [8], entre outros. Esses modelos consideram restrições de orçamento ou espaço físico. Outros modelos multiprodutos foram desenvolvidos posteriormente considerando diferentes aspectos como a substituição de produto [9], pedidos não atendidos [10] e seleção de fornecedores [11]. Muitos modelos multiprodutos como o de [12], [13] e [14] consideram também múltiplas restrições.

Outro fator importante considerado nos modelos de estoque é o tempo de ressuprimento do produto (lead time - LT). Os trabalhos [15] e [16] desenvolveram modelos que consideram o lead time variável. Outros aspectos também foram abordados nesses tipos de modelos. Por exemplo, [17] apresenta um modelo em que o lead time varia de acordo com o tamanho do lote e, [18] considera em seu modelo as vendas perdidas por indisponibilidade do fornecedor.

Diferentes padrões de demanda são abordados nos modelos de estoque encontrados na literatura, como os trabalhos de: [19], [20] e [21] que propuseram modelos com demanda fuzzy; [22] que abordaram a demanda sazonal em um modelo que considera também lead time variável e restrições de recurso e [23], que desenvolveram um modelo que considera os efeitos da publicidade e os esforços de vendas na demanda futura.

Os modelos de estoque em cadeias de suprimentos são também um tema recorrente na literatura nos últimos anos. Uma revisão de modelos que consideram múltiplos fornecedores foi apresentada por [24]. Já, em [25] desenvolveu-se um modelo para uma cadeia de suprimentos de produtos deterioráveis. Um modelo para produtos remanufaturados em cadeia de suprimentos foi proposto por [26]. Em [27] desenvolveu-se um modelo de controle de estoque com ressuprimento conjunto entre os elos da cadeia de suprimentos.

Ao longo do tempo, os modelos de estoque desenvolveramse no sentido de incorporar aspectos que representassem as diferentes características dos sistemas produtivos, podendo assim gerar soluções mais eficientes. Modelos de estoque 
multiprodutos que consideram múltiplas restrições são mais atuais, exigem soluções mais complexas e processamento computacional razoavelmente rápido, mostrando-se um promissor campo de pesquisa.

Os modelos de estoque multiprodutos apresentam diferentes formulações matemáticas devido às restrições que são levadas em consideração. Para representar esses modelos, os pesquisadores têm utilizado problemas de otimização não lineares e lineares. Em algumas das formulações não lineares são utilizadas técnicas de linearização para que possam ser utilizados métodos de otimização linear, devido à simplicidade de sua aplicação. A maioria dos trabalhos que utilizam formulações não lineares adota técnicas de otimização não determinística para resolvê-los como, por exemplo, os algoritmos genéticos. Esses métodos não são hábeis para garantir a otimalidade da solução encontrada.

Neste sentido, pretende-se formular, neste trabalho, o problema de estoque multiproduto como um problema de otimização não linear inteiro misto, baseado no trabalho de [12], o qual será resolvido por uma técnica de otimização determinística baseada no método Branch and Bound com o método de pontos interiores. Esse modelo decide qual a melhor opção entre o sistema de ponto de reposição e o de revisão periódica.

O modelo não linear inteiro misto, a ser apresentado neste trabalho, tem uma formulação mais simples que a do modelo linear proposto por [12], envolve uma quantidade menor de variáveis e restrições e, consequentemente, apresenta melhor desempenho computacional.

Este trabalho encontra-se dividido da seguinte maneira: na seção II definem-se os conceitos de sistemas de ponto de reposição e de revisão periódica; a proposta do modelo deste trabalho é apresentada na seção III; na seção IV é apresentada uma revisão de métodos de otimização, os quais serão utilizados neste trabalho; uma análise dos resultados numéricos obtidos, a partir de dois testes computacionais, com o modelo e o método escolhido para sua resolução é apresentada na seção $\mathrm{V}$ e, finalmente as conclusões e as perspectivas de trabalhos futuros são apresentadas na seção VI.

\section{PRINCIPAIS SISTEMAS DA GESTÃO DE ESTOQUES}

Os sistemas de gestão de estoques procuram determinar, basicamente, o momento do ressuprimento e a quantidade a ser ressuprida. Os autores de [28] apresentam o conceito de dois sistemas, tradicionalmente utilizados, que consideram itens com demanda independente uns dos outros: o sistema de ponto de reposição e o sistema de revisão periódica.

\section{A. Sistema de ponto de reposição}

O sistema de ponto de reposição é também conhecido na literatura como sistema de revisão contínua ou ciclo independente. Nesse sistema propõe-se o monitoramento contínuo do nível de estoque de cada item verificando-se a quantidade restante. Se a quantidade restante é menor que uma quantidade predeterminada, denominada ponto de reposição, libera-se a ordem de compra ou produção desse item numa quantidade definida (lote de ressuprimento). $\mathrm{O}$ ponto de reposição é determinado levando-se em consideração a quantidade que será consumida durante o tempo de ressuprimento (tempo entre a liberação da ordem de compra/produção e a chegada do item) e um estoque de segurança que considera as variações da demanda do item nesse período. A fig. 1 ilustra o funcionamento do sistema de ponto de reposição.

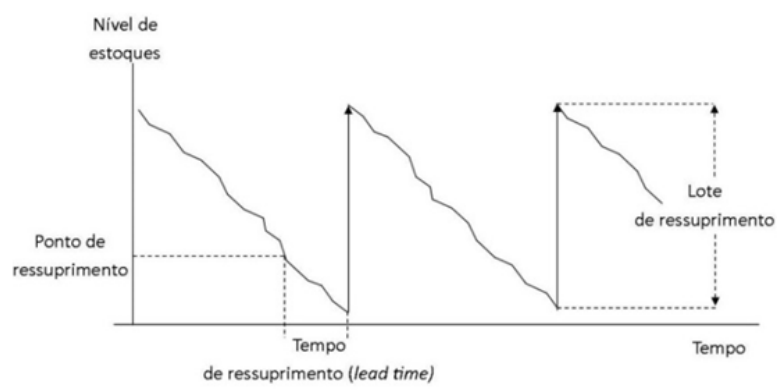

Figura 1. Sistema de ponto de reposição.

Para a utilização desse tipo de sistema é necessário definir dois parâmetros: o ponto de reposição e o tamanho do lote de ressuprimento. Este último é definido, basicamente, por uma modelagem matemática simples que calcula o tamanho de lote que gera o menor custo total do sistema de estoque. Nessa abordagem consideram-se os custos de armazenagem $(C A)$ e os custos de fazer pedidos $(C P)$ definidos da seguinte forma:

$C A=C_{e} \frac{X}{2}$

em que:

$C e$ : custo anual de estoque por unidade;

$X$ : quantidade pedida, e

$C P=C_{o} \frac{D}{X}$

em que:

Co: custo fixo de fazer pedido de ressuprimento; $D$ : demanda anual.

Considerando-se o custo total como a soma dos custos de armazenagem com os custos de fazer pedidos, tem-se que o tamanho de lote que gera o menor custo para a operação do sistema ocorre quando os custos de armazenagem e de fazer pedidos se igualam. A fig. 2 representa graficamente essa relação.

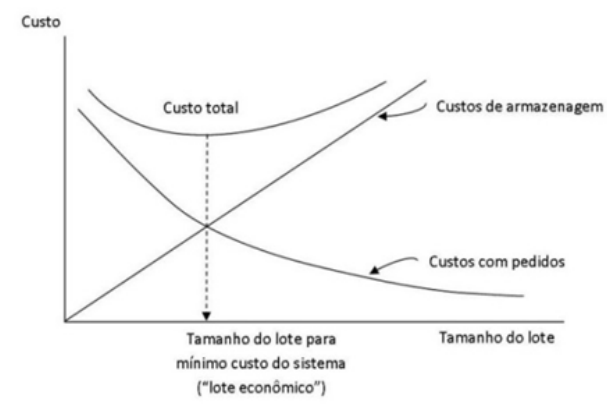

Figura 2. Custos totais do sistema de gestão de estoques da figura 1. 
O tamanho de lote para que os custos totais de manutenção do sistema sejam mínimos, denominado também de, lote econômico $\left(X_{E}\right)$, pode ser determinado através da equação que representa a igualdade dos custos de armazenagem e de fazer pedidos:

$$
C_{o} \frac{D}{X_{E}}=C_{e} \frac{X_{E}}{2}
$$

Portanto, o lote econômico é dado por:

$$
X_{\varepsilon}=\sqrt{\frac{2 D C_{O}}{C_{e}}}
$$

O ponto de ressuprimento $(P R)$ é definido pela multiplicação da demanda por unidade de tempo (D), pelo tempo de ressuprimento ( $L T)$, ambos na mesma unidade de tempo, somando-se a isso um estoque de segurança $\left(E_{\text {seg }}\right)$ como mostra a fórmula (5):

$P R=D L T+E_{\text {seg }}$

O estoque de segurança tem a finalidade de garantir que as variações da demanda que ocorrem de forma aleatória, não ocasionem falta do produto ou item.

\section{B. Sistema de revisão periódica}

O sistema de revisão periódica é conhecido também, na literatura, como ciclo fixo e, de acordo com [8], seu funcionamento é mais simples que o sistema de ponto de reposição. Neste sistema, o nível de estoque de cada item é verificado em períodos fixos (períodos de revisão) e ao final de cada período é definida a quantidade do item que deve ser ressuprida para que se atinja um nível predeterminado. Esse nível considera a demanda do item no período de revisão e um estoque de segurança. A fig. 3 ilustra o sistema de revisão periódica.

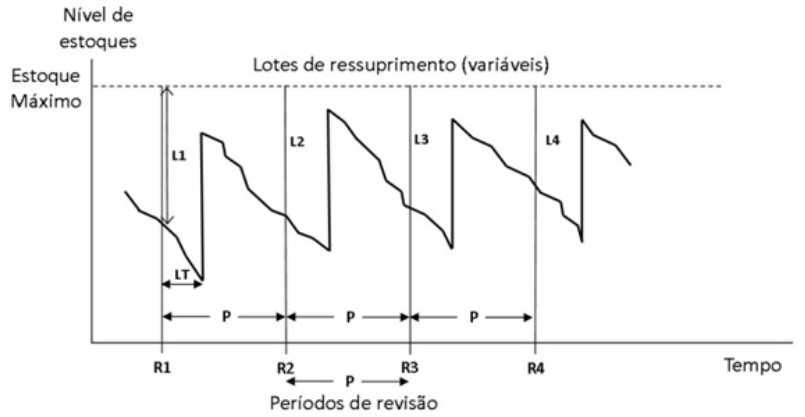

Figura 3. Sistema de revisão periódica.

A determinação da quantidade do item a ressuprir é realizada pela seguinte formulação:

$X=M-(E+Q P)$

em que:

$M$ : estoque máximo;

$E$ : estoque presente;
$Q P$ : quantidade pendente (já pedida) - eventual.

Como o nível máximo de estoque é definido pela fórmula:

$M=D(T+L T)+E_{s e g}$

em que:

$T$ : período entre pedidos;

$E_{\text {seg: }}$ estoque de segurança;

temos que:

$X=D(T+L T)+E_{s e g}-(E+Q P)$

As definições dos sistemas de gestão de estoques apresentados nesta seção, serão a base para o desenvolvimento do modelo proposto neste trabalho que será apresentado na seção III.

\section{MODELO NÃO LINEAR MULTIPRODUTO COM MÚLTIPLAS RESTRIÇÕES}

O modelo não linear inteiro misto multiproduto com múltiplas restrições, proposto neste trabalho, determina o menor custo entre o modelo de ponto de reposição e o de revisão periódica. Ele é baseado no modelo apresentado por [12], porém, a função objetivo utilizada está na forma não linear, assim como nos modelos utilizados por [8]. Esse modelo também considera múltiplas restrições e é formulado como um problema de otimização não linear inteiro misto, como segue:

$$
\begin{gathered}
\text { Minimizar } K(X)=\sum_{j=1}^{n}\left[\frac{C_{o j} D_{j}}{X_{j}}+c a c_{j}\left(\frac{X}{2}\right)\right] \\
\text { Sujeito } a: \sum_{j=1}^{n} w_{r j} X_{j} \leq M Z_{1}+B_{r} Z_{2}, \quad r=1,2, \ldots, v, \\
T_{j} \geq R Z_{1}, \quad j=1,2, \ldots, n, \\
T_{j} \leq R Z_{1}+Z_{2}, \quad j=1,2, \ldots, n, \\
T_{j} D_{j}-X_{j}=0, \quad j=1,2, \ldots, n, \\
Z_{1}+Z_{2}=1 \\
T_{j}, X_{j} \geq 0 \quad \forall j, \\
Z_{1}, Z_{2} \in\{0,1\}
\end{gathered}
$$

em que $K(X)$ é o custo anual total para todos os produtos.

Os parâmetros são:

$n$ : números de produtos;

$C_{o j}$ : custo de fazer o pedido para o produto $j$;

$D_{j}$ : demanda anual em unidades para o produto $j$;

$c_{j}$ : custo por unidade do produto $j$;

$c a$ : custo anual de carregamento de estoque;

$w_{r j}$ : quantidade do recurso $r$ consumida por uma unidade do produto $j$;

$r$ : restrições de recurso;

$B_{r}$ : disponibilidade do recurso $r$; 
$R: \operatorname{Min}\left\{\tau, \tau_{r} ; r=1,2, \ldots, v\right\}$;

$\tau$ : comprimento de ciclo ótimo para o problema irrestrito;

$\tau_{r}$ : comprimento de ciclo máximo permitido pela restrição do $r$-ésimo recurso;

$M$ : constante positiva muito grande;

e as variáveis são:

$X_{j}$ : quantidade pedida do produto $j$;

$T_{j}$ : tamanho do ciclo do produto $j$;

$\mathrm{Z1}, \mathrm{Z} 2$ : variáveis auxiliares: $\mathrm{Z1}=1$ e $\mathrm{Z} 2=0$, será utilizada a abordagem de revisão periódica; $Z 1=0$ e $Z 2=1$ será utilizada a abordagem de ponto de reposição.

$\mathrm{O}$ valor de $\tau$, denominado de comprimento de ciclo ótimo para o problema irrestrito, é calculado pela seguinte fórmula:

$$
\tau=\sqrt{\frac{2 \sum_{j=1}^{n} C_{o j}}{\sum_{j=1}^{n} c a c_{j} D_{j}}}
$$

o qual é obtido pela resolução do modelo de revisão periódica multiproduto irrestrito utilizado por [8] e o valor de $\tau_{r}$, denominado de comprimento de ciclo máximo permitido pela restrição do $r$-ésimo recurso, é dado por:

$$
\tau_{r}=\frac{2 B_{r}}{Q_{r}}
$$

com

$Q_{r}=\left[\sum_{j=1}^{n} w_{r j} D_{j}+\frac{\sum_{j=1}^{n}\left(w_{r j} D_{j}\right)^{2}}{\sum_{j=1}^{n} w_{r j} D_{j}}\right]$

Destaca-se que [12] em seu trabalho, linearizaram a função número de pedidos $N_{j}=\frac{D_{j}}{X_{j}}$. Desta forma, comparado ao modelo proposto em (9), o modelo de [12] apresenta uma formulação como um problema linear inteiro misto além de possuir um maior número de variáveis e restrições. No trabalho de [12] utilizou-se o método simplex.

$\mathrm{Na}$ seção IV serão apresentados o método Branch and Bound e o método de pontos interiores que serão utilizados na resolução do modelo proposto.

\section{MÉTODOS DE OTIMIZAÇÃO}

Apresenta-se nesta seção o método Branch and Bound e o método de pontos interiores os quais são utilizados neste trabalho para resolver o modelo proposto.

\section{A. Método Branch and Bound}

O método Branch and Bound (BB), também conhecido como método de avaliação e separação, foi originalmente proposto para problemas de programação linear inteira mista por [29]. No entanto, [30] utilizaram este método para problemas de programação não linear.

A estratégia do método consiste em uma enumeração sistemática dos candidatos a solução.

Divide-se o problema original com variáveis discretas em vários subproblemas contínuos, descartando-se conjuntos de soluções infrutíferas, a fim de se obter a solução ótima. Cada um destes subproblemas é resolvido por algum método de otimização contínuo. Neste trabalho adota-se o método de pontos interiores

Seja um problema inicial com variáveis discretas e contínuas (13):

$$
\begin{array}{ll}
\text { Minimizar } & f(x) \\
\text { sujeito a: } & h\left(x, y_{i}\right)=0 \\
& g\left(x, y_{i}\right) \leq 0 \\
& \underline{x} \leq x \leq \bar{x} \\
& y_{i} \in D_{y_{i}}, i=1,2, \ldots, n_{y}
\end{array}
$$

em que: $D_{y_{i}}, i=1,2, \ldots, n_{y}$ representa os conjuntos de valores discretos que $y_{i}$ deve assumir.

$O$ método $B B$ inicia-se resolvendo a relaxação contínua do problema (13) dada por (14):

$$
\begin{array}{ll}
\text { Minimizar } & f(x) \\
\text { sujeito a }: & h(x, y)=0 \\
& g(x, y) \leq 0 \\
& \underline{x} \leq x \leq \bar{x} \\
& \underline{y_{i}} \leq y_{i} \leq \overline{y_{i}}, \quad i=1,2, \ldots, n_{y}
\end{array}
$$

em que $\underline{y}_{i}=\operatorname{Min}\left\{D_{y_{i}}\right\}$ e $\overline{y_{i}}=\operatorname{Max}\left\{D_{y_{i}}\right\}, i=1,2, \ldots, n_{y^{\prime}}$.

Se na solução deste problema for obtido $y_{i} \in D_{y_{i}}$, para $i=1,2, \ldots, n y_{i}$, então a solução encontrada é ótima para o problema (13). Caso contrário, escolhe-se uma variável $y_{L}$ para ramificação e a região factível do problema (14) é dividida em duas subregiões (branch), fornecendo os dois subproblemas (15) e (16).

$\begin{array}{ll}\text { Minimizar } & f(x) \\ \text { sujeito } a: & h\left(x, y_{i}\right)=0 \\ & g\left(x, y_{i}\right) \leq 0 \\ & \underline{x} \leq x \leq \bar{x} \\ & \underline{y}_{i} \leq y_{i} \leq \overline{y_{i}} \\ & y_{L} \leq s\end{array}$


Minimizar $\quad f(x)$

sujeito a :

$$
\begin{aligned}
& h\left(x, y_{i}\right)=0 \\
& g\left(x, y_{i}\right) \leq 0 \\
& \underline{x} \leq x \leq \bar{x} \\
& \underline{y}_{i} \leq y_{i} \leq \bar{y}_{i} \\
& y_{L} \geq s
\end{aligned}
$$

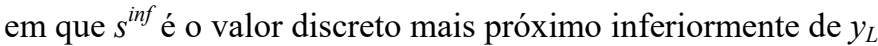
e $s^{s u p}$ é o valor discreto mais próximo superiormente de $y_{L}$.

Cada subproblema é um nó na árvore $B B$. A solução de cada subproblema fornece um limitante (bound) para a subregião. Se a solução do subproblema assume valores discretos para as variáveis discretas, então esta subregião não é mais explorada. Uma subregião em que a melhor solução discreta encontrada até o momento é melhor que seu limitante, é descartada, caso contrário é explorada recursivamente. Desta forma, uma árvore de subproblemas é criada e o método termina quando não há mais subregiões a serem exploradas

\section{B. Método de pontos interiores}

Um dos primeiros métodos de pontos interiores foi denominado de método de barreira e apresentado por [31], para a função barreira logarítmica e por [32] para a função barreira inversa. Este método foi popularizado por [33], em 1984, quando apresentou seu método projetivo para programação linear.

Após isso, muitos pesquisadores passaram a aprimorar o método de [33] e uma versão do método de pontos interiores para a programação não linear foi apresentada por [34].

A seguir apresenta-se uma descrição do método de pontos interiores, proposto por [35], o qual encontra-se implementado no pacote Knitro e é utilizado neste trabalho.

Seja o problema geral de otimização não linear representado como segue:

$\begin{array}{ll}\text { Minimizar } & f(x) \\ \text { sujeito a: } & h(x)=0 \\ & g(x) \leq 0\end{array}$

em que:

$x \in \mathbb{R}^{n}, f: \mathbb{R}^{n} \rightarrow \mathbb{R}, g: \mathbb{R}^{n} \rightarrow \mathbb{R}^{m}, h: \mathbb{R}^{n} \rightarrow \mathbb{R}^{l}$

e $f, g$ e $h$ são classes de $C^{2}$.

Ao adicionar variáveis de folga não negativas às restrições de desigualdade de (17), estas são transformadas em restrições de igualdade. Desta forma, as restrições de não negatividade das variáveis de folga são incorporadas na função objetivo através da função de barreira logarítmica de [31] e dá origem ao problema de barreira (18).

$$
\begin{array}{ll}
\text { Minimizar } & f(x)-\mu \sum_{i=1}^{m} \ln \left(s_{i}\right) \\
\text { sujeito a: } & h(x)=0 \\
& g(x)+s=0
\end{array}
$$

em que: $s=\left(s_{1}, s_{2}, \ldots, s_{m}\right)^{t}$ é o vetor das variáveis de folga, $\operatorname{com} s_{i} \in \mathbb{R}, i=1,2, \ldots, m$ e $\mu>0$ é denominado parâmetro de barreira.

Associa-se ao problema de barreira (18) a seguinte função lagrangiana:

$$
\begin{aligned}
L(x, s, \lambda, \mu)= & f(x)-\mu \sum_{i=1}^{m} \ln \left(s_{i}\right)+\lambda_{h}^{T} h(x) \\
& +\lambda_{g}^{T}(g(x)+s)
\end{aligned}
$$

em que: $\lambda_{h} \in \mathbb{R}^{l}$ e $\lambda_{g} \in \mathbb{R}^{m}$ são denominados de vetores dos multiplicadores de Lagrange. A ideia do método é resolver uma sequência de problemas irrestritos cuja função objetivo é dada por (19), para um parâmetro de barreira fixo $\mu_{k}$, o qual será atualizado a cada iteração.

Para a solução desses problemas irrestritos, aplica-se a condição necessária de primeira ordem na função lagrangiana (19) e obtém-se um sistema não linear. Esse sistema é resolvido pelo método de Newton o qual determina as direções de busca $d_{x}, d_{y}, d_{\lambda_{h}}, d_{\lambda_{g}}$ e uma nova solução é obtida por:

$$
\begin{aligned}
& x^{k+1}=x^{k}+\alpha_{p} d_{x}^{k}, \\
& s^{k+1}=s^{k}+\alpha_{p} d_{s}^{k} \\
& \lambda_{h}^{k+1}=\lambda_{h}{ }^{k}+\alpha_{d} d^{k} \lambda_{h} \\
& \lambda_{g}{ }^{k+1}=\lambda_{g}{ }^{k}+\alpha_{d} d^{k} \lambda_{g}
\end{aligned}
$$

em que $\alpha_{p}$ e $\alpha_{d}$ são os tamanhos de passos primais e duais, respectivamente, e seus cálculos podem ser encontrado em [36].

O parâmetro de barreira é atualizado utilizando-se a regra $\mu_{k+1}=\frac{\mu_{k}}{\beta}, \beta>1$ e o processo é repetido até que um critério de convergência seja satisfeito.

\section{RESULTADOS COMPUTACIONAIS}

Neste capítulo apresentam-se os resultados computacionais obtidos. Os testes foram divididos em duas etapas. A $1^{\text {a }}$ etapa de testes foi realizada utilizando uma instância baseada no trabalho de [12], e foi realizada com o objetivo de comparar o modelo linear inteiro misto proposto por este autor com o modelo proposto neste trabalho e apresentado na seção III. A $2^{\mathrm{a}}$ etapa de testes, também realizada com instâncias baseadas em [12], tem como objetivo testar a eficácia do modelo proposto e da abordagem de resolução escolhida, para problemas com diferentes dimensões.

Para a resolução dos modelos foram utilizados alguns métodos de otimização. Para a resolução do modelo linear 
inteiro misto apresentado por [12] foi utilizado o algoritmo Branch and Cut juntamente com o algoritmo simplex/dual simplex que estão implementados, no pacote de otimização CPLEX. Para a resolução do modelo proposto neste trabalho foi utilizado um algoritmo baseado no algoritmo Branch and Bound com o método de pontos interiores, o qual encontra-se implementado no pacote de otimização KNITRO.

Todos os testes foram realizados em um computador Intel Core i7, com 8GB de memória RAM e 1TB de HD.

\section{A. PRIMEIRA ETAPA DE TESTES}

A instância utilizada neste teste possui dez produtos, $j=1$, $2, \ldots, 10$, e cinco restrições de recurso, $r=1,2, \ldots, 5$. A tabela I apresenta os parâmetros referentes aos produtos.

TABELA I

PARÂMETROS, REFERENTES AOS PRODUTOS, UTILIZADOS NA $1^{\text {a }}$ ETAPA DE TESTES

\begin{tabular}{|c|c|c|c|}
\hline $\boldsymbol{j}$ & $\begin{array}{c}\text { Demanda } \\
\text { anual do } \\
\text { produto } \boldsymbol{j} \\
\left(\boldsymbol{D}_{\boldsymbol{j}}\right)\end{array}$ & $\begin{array}{c}\text { Custo anual de } \\
\text { estoque por unidade } \\
\text { do produto } \boldsymbol{j} \\
\left(\boldsymbol{c a}_{\boldsymbol{~}} \boldsymbol{j}\right)\end{array}$ & $\begin{array}{c}\text { Custo de fazer } \\
\text { pedidos do produto } \boldsymbol{j} \\
\left(\boldsymbol{C}_{\boldsymbol{o} \boldsymbol{j}}\right)\end{array}$ \\
\hline 1 & 354 & 50 & 221 \\
2 & 743 & 34 & 239 \\
3 & 563 & 88 & 248 \\
4 & 486 & 78 & 162 \\
5 & 320 & 34 & 199 \\
6 & 764 & 70 & 129 \\
7 & 943 & 34 & 277 \\
8 & 624 & 18 & 151 \\
9 & 272 & 12 & 378 \\
10 & 806 & 40 & 129 \\
\hline
\end{tabular}

Os parâmetros referentes às restrições são apresentados na tabela II.

TABELA II

PARÂMETROS, REFERENTES ÀS RESTRIÇÕES, UTILIZADOS NA $1^{\text {a }}$ ETAPA DE TESTES

\begin{tabular}{|c|c|c|c|c|c|}
\hline \multicolumn{6}{|c|}{ Disponibilidade do recurso r $\left(\boldsymbol{B}_{r}\right)$} \\
\hline \multicolumn{7}{|c|}{$r=1$} & $r=2$ & $r=3$ & $r=4$ & $r=5$ \\
\hline \multicolumn{7}{|c|}{ Quantidade do recurso r consumida por uma unidade do produto } \\
$\mathbf{5}\left(\boldsymbol{w}_{\boldsymbol{r}}\right)$
\end{tabular}

Ressalta-se que, devido à linearização da função número de pedidos $N_{j}$, o modelo apresentado por [12] tem dimensões, para esse teste, muito maiores que às do modelo proposto neste trabalho. Enquanto que o modelo de [12] tem 640 variáveis e 662 restrições, o modelo proposto tem 30 variáveis e 47 restrições.

Alguns resultados como, valores ótimos, quantidade pedida e tamanho do ciclo, nas soluções obtidas, são apresentados na tabela III.
TABELA III

VALORES ÓTIMOS $-1^{\text {a }}$ ETAPA DE TESTES

\begin{tabular}{|c|c|c|c|c|c|}
\hline & \multicolumn{3}{|c|}{ Modelo de Haksever [12] } & \multicolumn{2}{|c|}{ Modelo proposto } \\
\hline Prod. (j) & $X_{j}$ & $N_{j}$ & $T_{j}$ & $X_{j}$ & $T_{j}$ \\
\hline 1 & 44,206 & 8,035 & 0,125 & 44,206 & 0,125 \\
\hline 2 & 92,782 & 8,036 & 0,125 & 92,782 & 0,125 \\
\hline 3 & 70,305 & 8,063 & 0,125 & 70,305 & 0,125 \\
\hline 4 & 60,689 & 8,048 & 0,125 & 60,689 & 0,125 \\
\hline 5 & 39,96 & 8,11 & 0,125 & 39,96 & 0,125 \\
\hline 6 & 95,404 & 8,07 & 0,125 & 95,405 & 0,125 \\
\hline 7 & 117,757 & 8,055 & 0,125 & 117,757 & 0,125 \\
\hline 8 & 77,922 & 8,026 & 0,125 & 77,922 & 0,125 \\
\hline 9 & 33,966 & 8,057 & 0,125 & 33,966 & 0,125 \\
\hline 10 & 100,649 & 8,123 & 0,125 & 100,649 & 0,125 \\
\hline$K(N, X)$ & \multicolumn{3}{|c|}{34162,23} & \multicolumn{2}{|c|}{34162,23} \\
\hline
\end{tabular}

Os dados da tabela III mostram que os valores ótimos encontrados para os modelos testados são iguais. O sistema de estoque apontado como melhor opção por ambos os foi o de revisão periódica.

Os resultados indicam que o modelo e os métodos de resolução, propostos neste trabalho, podem ser utilizados em problemas de estoque multiprodutos.

\section{B. Segunda Etapa de Testes}

A Segunda Etapa de Testes foi realizada com 24 instâncias diferentes com variações na quantidade de produtos e restrições de recurso. Os parâmetros do modelo foram gerados utilizando-se o gerador de números aleatórios do pacote Excel, de acordo com os limites propostos por [12], exceto para o parâmetro disponibilidade do recurso $r(\mathrm{Br})$ que foi definido pelos autores. Os 12 primeiros problemas referem-se à demanda cujo limite inferior é adotado como 200 e o superior como 1000 , enquanto que, os 12 últimos referem-se à demanda cujo limite superior foi alterado para 2000. Para cada conjunto de demanda foram elaborados problemas com 5, 10, 20 e 30 produtos, sendo que, para cada quantidade de produtos foram definidos 3 , 4 e 5 restrições de recursos.

A tabela IV apresenta os valores adotados para os parâmetros do modelo em relação às quantidades e limites inferiores e superiores dos parâmetros em que foram gerados os números aleatórios.

TABELA IV

VALORES, LIMITES INFERIOR E SUPERIOR DOS PARÂMETROS UTILIZADOS UTILIZADOS NA $2^{\mathrm{a}}$ ETAPA DE TESTES

\begin{tabular}{|c|c|c|}
\hline Parâmetros & Definição & Valores/limites \\
\hline$n$ & números de produtos & $5,10,20,30$ \\
\hline$D_{j}$ & $\begin{array}{c}\text { demanda anual em unidades para o } \\
\text { produto } j\end{array}$ & $\begin{array}{c}\text { inferior: } 200 \\
\text { superior: } 1000 / 2000\end{array}$ \\
\hline$C_{o j}$ & $\begin{array}{c}\text { custo de fazer o pedido para o } \\
\text { produto } j\end{array}$ & $\begin{array}{c}\text { inferior: } 100 \\
\text { superior: } 400\end{array}$ \\
\hline$c a c_{j}$ & $\begin{array}{c}\text { custo anual de carregamento por } \\
\text { unidade do produto } j\end{array}$ & $\begin{array}{c}\text { inferior: } 10 \\
\text { superior: } 100\end{array}$ \\
\hline$r$ & restrições de recurso & $3,4,5$ \\
\hline$w_{r j}$ & $\begin{array}{c}\text { quantidade do recurso } r \text { consumida } \\
\text { por uma unidade do produto } j\end{array}$ & $\begin{array}{c}\text { inferior: } 10 \\
\text { superior: } 100\end{array}$ \\
\hline
\end{tabular}

A tabela $\mathrm{V}$ apresenta o limite inferior e o superior da disponibilidade do recurso $r,(\mathrm{Br})$, de acordo com a quantidade de produtos do problema e os limites da demanda anual. 
TABELA V

LIMITES INFERIOR E SUPERIOR DA DISPONIBILIDADE DO RECURSO $r$ UTILIZADOS NA $2^{\text {a }}$ ETAPA DE TESTES

\begin{tabular}{|c|c|c|}
\hline $\begin{array}{c}\text { Limites inferior } \\
\text { e superior da } \\
\text { demanda }\end{array}$ & $\begin{array}{c}\text { Número de } \\
\text { produtos }\end{array}$ & $\begin{array}{c}\text { Limites inferior e superior } \\
\text { da disponibilidade do } \\
\text { recurso } \boldsymbol{r}\left(\boldsymbol{B}_{\boldsymbol{r}}\right)\end{array}$ \\
\hline \multirow{3}{*}{$200-1000$} & 5 & $12500-25000$ \\
\cline { 2 - 3 } & 10 & $25000-50000$ \\
\cline { 2 - 3 } & 20 & $50000-100000$ \\
\hline \multirow{3}{*}{$200-2000$} & 30 & $75000-150000$ \\
\cline { 2 - 3 } & 5 & $12500-50000$ \\
\cline { 2 - 3 } & 10 & $25000-100000$ \\
\cline { 2 - 3 } & 20 & $50000-200000$ \\
\hline
\end{tabular}

As dimensões das instâncias geradas referentes ao número de produtos, restrições de recurso, variáveis e restrições do modelo são apresentadas na tabela VI.

TABELA VI

DIMENSÕES DOS PROBLEMAS DA $2^{\text {a }}$ ETAPA DE TESTES

\begin{tabular}{|c|c|c|c|c|}
\hline Problema & $\begin{array}{c}\mathrm{N}^{\circ} \text { de } \\
\text { produtos }\end{array}$ & $\begin{array}{c}N^{\circ} \text { de restrições } \\
\text { de recurso }\end{array}$ & $\begin{array}{c}\mathrm{N}^{\circ} \text { de } \\
\text { variáveis }\end{array}$ & $\begin{array}{l}\mathrm{N}^{\circ} \text { de restrições } \\
\text { do modelo }\end{array}$ \\
\hline 1 & 5 & 3 & 17 & 26 \\
\hline 2 & 5 & 4 & 18 & 29 \\
\hline 3 & 5 & 5 & 19 & 32 \\
\hline 4 & 10 & 3 & 27 & 41 \\
\hline 5 & 10 & 4 & 28 & 44 \\
\hline 6 & 10 & 5 & 29 & 47 \\
\hline 7 & 20 & 3 & 47 & 71 \\
\hline 8 & 20 & 4 & 48 & 74 \\
\hline 9 & 20 & 5 & 49 & 77 \\
\hline 10 & 30 & 3 & 67 & 101 \\
\hline 11 & 30 & 4 & 68 & 104 \\
\hline 12 & 30 & 5 & 69 & 107 \\
\hline 13 & 5 & 3 & 17 & 26 \\
\hline 14 & 5 & 4 & 18 & 29 \\
\hline 15 & 5 & 5 & 19 & 32 \\
\hline 16 & 10 & 3 & 27 & 41 \\
\hline 17 & 10 & 4 & 28 & 44 \\
\hline 18 & 10 & 5 & 29 & 47 \\
\hline 19 & 20 & 3 & 47 & 71 \\
\hline 20 & 20 & 4 & 48 & 74 \\
\hline 21 & 20 & 5 & 49 & 77 \\
\hline 22 & 30 & 3 & 67 & 101 \\
\hline 23 & 30 & 4 & 68 & 104 \\
\hline 24 & 30 & 5 & 69 & 107 \\
\hline
\end{tabular}

A tabela VII apresenta o tipo de sistema, valor ótimo e tempo médio de processamento, nas soluções ótimas obtidas para as instâncias.

TABELA VII

RESULTADOS DA $2{ }^{\text {a }}$ ETAPA DE TESTES

\begin{tabular}{|c|c|c|c|}
\hline Problema & Tipo de sistema & Valor ótimo & $\begin{array}{c}\text { Tempo médio de } \\
\text { processamento (s) }\end{array}$ \\
\hline 1 & Revisão periódica & $24.993,855$ & 0,096 \\
2 & Ponto de reposição & $14.531,690$ & 0,045 \\
3 & Ponto de reposição & $15.113,446$ & 0,087 \\
4 & Revisão periódica & $42.252,515$ & 0,078 \\
5 & Ponto de reposição & $39.902,828$ & 0,103 \\
6 & Ponto de reposição & $36.261,577$ & 0,105 \\
7 & Ponto de reposição & $73.044,683$ & 0,086
\end{tabular}

\begin{tabular}{|c|c|c|c|}
8 & Ponto de reposição & $87.544,727$ & 0,078 \\
9 & Ponto de reposição & $78.180,179$ & 0,105 \\
10 & Ponto de reposição & $120.247,578$ & 0,112 \\
11 & Ponto de reposição & $126.495,946$ & 0,157 \\
12 & Revisão periódica & $111.717,585$ & 0,131 \\
13 & Ponto de reposição & $33.635,540$ & 0,076 \\
14 & Ponto de reposição & $17.522,394$ & 0,055 \\
15 & Ponto de reposição & $15.823,868$ & 0,061 \\
16 & Revisão periódica & $55.492,840$ & 0,110 \\
17 & Ponto de reposição & $53.146,761$ & 0,067 \\
18 & Ponto de reposição & $54.904,470$ & 0,092 \\
19 & Ponto de reposição & $96.718,847$ & 0,126 \\
20 & Revisão periódica & $95.569,293$ & 0,116 \\
21 & Revisão periódica & $107.114,998$ & 0,150 \\
22 & Ponto de reposição & $146.450,862$ & 0,114 \\
23 & Ponto de reposição & $148.771,248$ & 0,124 \\
24 & Ponto de reposição & $157.876,610$ & 0,156 \\
\hline
\end{tabular}

O modelo proposto neste trabalho e o método de resolução adotado, Branch and Bound com método de pontos interiores, mostraram-se eficaz na determinação da solução dos problemas propostos. O tempo médio de processamento para obtenção das soluções dos problemas foi razoavelmente rápido, entre $0,045 \mathrm{~s}$ e $0,157 \mathrm{~s}$, como mostra a tabela VII. Mesmo com a variação do número de produtos, de 5 a 30 , e de restrições de recurso, de 3 a 5 , dos problemas, o tempo médio de processamento entre eles não variou expressivamente.

Ao compararmos o tempo médio de processamento com o obtido no trabalho de [12] destacamos que os modelos resolvidos com o método de Branch and Bound com método de pontos interiores levaram um tempo de processamento menor. Essa diferença é maior nos problemas de maior dimensão, com 20 e 30 produtos.

\section{CONCLUSÕES E TRABALHOS FUTUROS}

Neste trabalho propôs-se uma investigação sobre a modelagem e a resolução de problemas de otimização em sistemas de estoque multiproduto. Um novo modelo o qual aponta a melhor opção entre o conceito de ponto de reposição e o de revisão periódica, foi proposto. Este modelo foi formulado como um problema de otimização não linear inteiro misto. Para a determinação do novo modelo utilizou-se o trabalho de [12]. Destaca-se que, o modelo aqui proposto difere do trabalho [12] por trabalhar com a função custo na forma não linear, a qual se aproxima mais da situação real.

Para a resolução deste modelo utilizou-se uma abordagem que é baseada no método de Branch and Bound com o método de ponto interiores. Da revisão bibliográfica dos trabalhos que abordam o tema de modelos de estoque multiprodutos com múltiplas restrições, observou-se que são raros os trabalhos, que ao formular o modelo de estoque multiprodutos como um problema de otimização não linear, o resolvem através de técnicas de otimização determinística como é o caso do método abordado nesse trabalho. A grande maioria dos trabalhos trabalha com a linearização das restrições não lineares, ou com algoritmos não determinísticos para determinar a sua solução, como algoritmos genéticos. 
Testes computacionais foram realizados com o modelo proposto e os métodos de resolução escolhidos com diferentes objetivos.

$\mathrm{Na} 1^{\text {a }}$ Etapa de Testes, o objetivo foi comparar os resultados obtidos pelo modelo proposto neste trabalho, apresentado na seção III, com os resultados obtidos por [12] a fim de verificar a aplicabilidade dos métodos de otimização não lineares utilizados no modelo proposto em problemas de sistemas com múltiplas restrições. Os resultados obtidos indicam que os problemas de gestão de estoque multiproduto com múltiplas restrições podem ser resolvidos pelo modelo não linear proposto neste trabalho através de um método de otimização determinístico, baseado no método de Branch and Bound com o método de pontos interiores. Além disso, neste modelo não linear tem-se a vantagem de se trabalhar com um problema de dimensão menor que o modelo de [12] e representa-se a função custo, de maneira mais próxima à realidade.

$\mathrm{Na} 2^{\mathrm{a}}$ Etapa de Testes, o objetivo foi testar a eficácia do modelo proposto e da abordagem de resolução escolhida para instâncias com diferentes dimensões. Nesta etapa, foram resolvidas 24 instâncias com diferentes dimensões. Para isso, foram gerados randomicamente os dados para os problemas utilizando o software Excel. Os resultados demonstram a potencialidade do modelo proposto e do método de resolução eleito.

Neste sentido contribui-se com a área de gestão de estoques apresentando um modelo mais próximo à realidade das indústrias e com a área de otimização ao verificar-se que é possível resolver o modelo proposto através de um método determinístico. Além disso, o modelo proposto, quando implementado, apresenta um bom desempenho computacional, importante para a tomada de decisão na gestão de estoques.

Uma das limitações apresentadas ao nosso método é que ele determina soluções inteiras somente para as variáveis binárias enquanto que as demais variáveis assumem valores reais. Desta forma, em trabalhos futuros pretende-se estabelecer uma abordagem de resolução alternativa que produza resultados eficazes reais para o modelo da seção III. Além disso, existe também a possibilidade de aprimorar o modelo ao incluir outras restrições e variáveis específicas, mais próximas da realidade. Outra análise, que ainda pode ser realizada futuramente, é a de verificar qual é a sensibilidade do modelo em relação à mudança de ponto de revisão para revisão periódica e vice -versa.

\section{AGRADECIMENTOS}

Os autores agradecem ao Conselho Nacional de Desenvolvimento Científico e Tecnológico ( $\mathrm{CNPq}$ ) pelo auxílio financeiro concedido através dos processos 448645/2014-9 e 309588/2013-8 (bolsista CNPq).

\section{REFERÊNCIAS}

[1] N. Slack, S. Chambers, R. Johnston. Administração da produção. 3. ed. São Paulo: Atlas, 2009.

[2] D. P. Koumanakos. The effect of inventory management on firm performance. International Journal of Productivity and Performance Management, vol. 57, no. 5, pp. 355-369, 2008.
[3] G. Vastag, D. C. Whybark. Inventory management - Is there a knock-on effect? International journal of production economics, vol. 93-94, pp. 129$138,2005$.

[4] J. A. Parsons. Multi-product lot size determination when certain restrictions are active. Journal of Industrial Engineering, vol. 17, pp. 360-365, 1966.

[5] S. K. Goyal. Optimum ordering policy for a multi-item single supplier system. Operational Research Quarterly, vol. 25, pp. 293-298, 1974.

[6] E. Page, R. J. Paul. Multi-product inventory situations with one restriction. Operational Research Quarterly, vol. 27, no. 4, pp. 815-834, 1976.

[7] K. Zoller. Deterministic multi-item inventory systems with limited capacity. Management Science, vol. 24, pp. 451-455, 1977.

[8] M. Rosenbaltt. Multi-item inventory system with budgetary constraint: a comparison between the Lagrangian and the fixed cycle approach. International journal of production research, vol. 19, no. 4, pp. 331-339, 1981

[9] Y. Bassok, R. Anupindi, R. Akella. Single-period multiproduct inventory models with substitution. Operations research, vol. 47, no. 4, pp. 632-642, 1999.

[10] B. Ghalebsaz-Jeddi, B. C. Shultes, R. A. Haji. multi-product continuous review inventory system with stochastic demand, backorders, and a budget constraint. European journal of operational research, vol. 158, pp. 456-469, 2004.

[11] J. Rezaei, M. Davoodi. A deterministic, multi-item inventory model with supplier selection and imperfect quality. Applied mathematical modelling, vol. 32, pp. 2106-2116, 2008.

[12] C. Haksever, J. Moussourakis. A model for optimizing multi-product inventory systems with multiple constraints. International journal of production economics, vol. 97, pp. 18-30, 2005.

[13] A. A. Taleizadeh, M. Aryanezhad, S. T. A. Niaki. Optimizing multiproduct multi-constraint inventory control systems with stochastic replenishments. Journal of applied sciences, vol. 8, no. 7, pp. 1228-1234, 2008.

[14] S. H. R. Pasandideh, S. T. A. Niaki, A. R. A. Nia. Genetic algorithm for vendor managed inventory control system of multi-product multi-constraint economic order quantity model. Expert Systems with Applications, vol. 38, pp. 2708-2716, 2011.

[15] M. Hariga, M. Ben-Daya. Some stochastic inventory models with deterministic variable lead time. European journal of operational research, vol. 113, pp. 42-51, 1999.

[16] J. H. Bookbinder, M. Cakanyildirim. Random lead times and expedited orders in $(\mathrm{Q}, \mathrm{r})$ inventory systems. European journal of operational research, vol. 115, pp. 300-313, 1999.

[17] M. Çakanyildirim, J. H. Bookbinder, Y. Gerchak. Continuous review inventory models where random lead time depends on lot size and reserved capacity. International journal of production economics, vol. 68, pp. 217-228, 2000.

[18] E. Mohebbi. Supply interruptions in a lost-sales inventory system with random lead time. Computers \& operations research, vol. 30, pp. 411-426, 2003.

[19] C. H. Hsieh. Optimization of fuzzy production inventory models. Information sciences, vol. 146, pp. 2940, 2002.

[20] C. Kao, W. Hsu. A single-period inventory model with fuzzy demand. Computers and mathematics with applications, vol. 43, pp. 841-848, 2002.

[21] P. Dutta, D. Chakraborty, A. R. Roy. A single-period inventory model with fuzzy random variable demand. Mathematical and computer modelling, vol. 41, pp. 915-922, 2005

[22] K. K. Chen, C. Chang. A seasonal demand inventory model with variable lead time and resource constraints. Applied mathematical modelling, vol. 31, pp. 2433-2445, 2007.

[23] S. S. Sana, K. S. Chaudhuri. An inventory model for stock with advertising sensitive demand. IMA Journal of management mathematics, vol. 19 , pp. 51-62, 2008.

[24] S. Minner. Multiple-supplier inventory models in supply chain management - a review. International journal of production economics, vol. 81-82, pp. 265-279, 2003.

[25] H. Rau, M. Wu, H. Wee. Integrated inventory model for deteriorating items under a multi-echelon supply chain environment. International journal of production economics, vol. 86, pp. 155-168, 2003.

[26] S. Chung, H. Wee, P. Yang. Optimal policy for a closed-loop supply chain inventory system with remanufacturing. Mathematical and computer modelling, vol. 48, pp. 867-881, 2008.

[27] W. Zhou, L. Chen, H. Ge. A multi-product multi-echelon inventory control model with joint replenishment strategy. Applied mathematical modelling, vol. 37, pp. 2039-2050, 2013. 
[28] H. Corrêa, I. Gianesi, M. Caon. Planejameto, programação e controle da produção. 5. ed. São Paulo: Atlas, 2007.

[29] A. H. Land, A. G. Doig. An Automatic Method of Solving Discrete Programming Problems. Econometrica, vol. 28, no. 3, pp. 497-520, 1960.

[30] O. K. Gupta, A. Ravindran. Branch and bound experiments in convex nonlinear integer programming. Management science, INFORMS, vol. 31, no. 12, pp. 1533-1546, 1985.

[31] K. R. Frisch. The logarithmic Potential Method of Convex Programming, Memorandum, University Institute of Economics, Oslo, Norway, 1955.

[32] C. W. Carrol. The Created Response Surface Technique for Optimizing Nonlinear Restrained Systems, Operations research, vol. 9, pp. 169-184, 1961.

[33] N. Karmarkar. A new polynomial-time algorithm for linear programming. In Proceedings of the sixteenth annual ACM symposium on Theory of computing, pp. 302-311, 1984.

[34] A. S. El-Bakry, R. A. Tapia, T. Tsuchiya, Y. Zhang. On the Formulation and Theory of the Newton Interior-Point Method for Nonlinear Programming. Journal of Optimization Theory and Applications, vol. 89, no.3, pp. 507-541, 1996.

[35] R. A. Waltz, J. L. Morales, J. Nocedal, D. Orban. An interior algorithm for nonlinear optimization that combines line search and trust region steps, Mathematical Programming Series A, vol. 107, pp. 391-408, 2006.

[36] R. H. Byrd, M. E. Hribar, J. Nocedal. An interior point algorithm for large scale nonlinear programming, Siam Journal on Optimization, vol. 9, n. 4 pp. 877-900, 1999.

[37] J. Nocedal, A. Wächter, R. A. Waltz. Adaptive barrier strategies for nonlinear interior methods, Technical Report RC 23563, IBM Watson Research Center, Yorktown Heights, NY, USA, 2008.

[38] E. M. Soler, V. A. Sousa, G. R. M. Costa. A modified Primal-Dual Logarithmic-Barrier Method for solving the Optimal Power Flow problem with discrete and continuous control variables, European Journal of Operational Research, vol. 222, no. 3 pp. 616-622, 2012

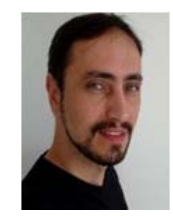

Álvaro De Martino Lourenção, é graduado em Engenharia de Produção pela Universidade Federal de São Carlos UFSCar, São Carlos- SP, em 2006, tendo obtido o título de Mestre em Engenharia de Produção da Universidade Estadual Paulista - UNESP, Bauru-SP, em 2016. Atualmente desenvolve pesquisas na área de otimização, atuando principalmente nos seguintes temas: programação não linear e planejamento da produção.

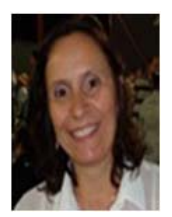

Edméa Cássia Baptista, é graduada em Matemática pela Universidade Estadual Paulista - UNESP - SP, em 1988, tendo obtido o título de mestre em Ciências da Computação, em 1993, título de doutora em Engenharia Elétrica, em 2001, ambos pela Universidade de São Paulo - USP. Obteve a Livre Docência pela Universidade Estadual Paulista UNESP, em 2013. Atualmente é Professora Adjunta do Departamento de Matemática da UNESP, em Bauru e docente permanente dos Programas de Pós-graduação em Engenharia de Produção e Engenharia Elétrica, também da UNESP, onde desenvolve pesquisas na área de otimização, atuando principalmente nos seguintes temas: programação não linear, problema de fluxo de potência ótimo e planejamento da produção.

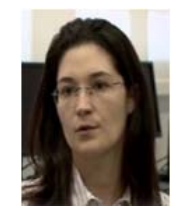

Edilaine Martins Soler, é graduada em Matemática pela Universidade de São Paulo - USP, em 2007, tendo obtido o título de mestre em Ciência da Computação e Matemática Computacional, em 2008, e título de doutora em Engenharia Elétrica, em 2011, ambos pela Universidade de São Paulo USP. Atualmente é Professora Assistente da Universidade Estadual Paulista - UNESP - SP e docente permanente dos Programas de Pós-graduação em Engenharia de Produção e Engenharia Elétrica, também da UNESP, onde desenvolve pesquisas na área de otimização, atuando principalmente nos seguintes temas: programação não linear, variáveis discretas e problema de fluxo de potência ótimo.

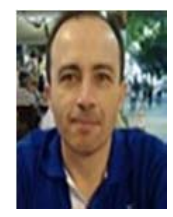

Fernando Bernardi de Souza, é graduado em Engenharia de Produção Mecânica pela Universidade de São Paulo - USP em 1994, tendo obtido o título de mestre em Engenharia Mecânica em 1997 e título de doutor em Engenharia Mecânica em 2001, ambos pela Universidade de São Paulo USP. Obteve a Livre Docência pela Universidade Estadual Paulista - UNESP em 2011. Atualmente é Professor Adjunto do Departamento de Engenharia de Produção da Faculdade de Engenharia de Bauru - UNESP e docente permanente do Programa de Pós-graduação em Engenharia de Produção, também da UNESP, onde desenvolve pesquisas na área de Engenharia de Produção, atuando principalmente nos seguintes temas: sistemas de planejamento e controle da produção, gestão da cadeia de suprimentos, teoria das restrições e manufatura enxuta.

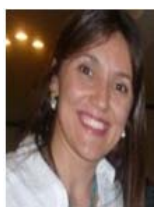

Adriana Cristina Cherri, é graduada em Matemática pela Universidade Estadual Paulista - UNESP - SP, em 2001, tendo obtido o título de mestre em Ciências da Computação e Matemática Computacional, em 2006, e Doutorado em Ciências da Computação e Matemática Computacional, em 2009, ambos pela Universidade de São Paulo - USP Atualmente é Professora Assistente do Departamento de Matemática da UNESP, em Bauru, e docente permanente do Programa de Pós-graduação em Engenharia de Produção, também da UNESP, onde desenvolve pesquisas na área de otimização, atuando principalmente nos seguintes temas: programação linear inteira, problemas de corte e empacotamento, geração de colunas e modelagem matemática. 